\title{
CD30-positive cutaneous lymphoma: report of four cases with an emphasis on clinicopathological correlations*
}

\author{
Thiago Jeunon de Sousa Vargas ${ }^{1}$ \\ Yung Bruno de Mello Gonzaga ${ }^{1,2}$
}

\author{
Samira Barroso Jorge ${ }^{1}$
}

DOI: http:/ / dx.doi.org/10.1590/abd1806-4841.20174807

\begin{abstract}
The classification of cutaneous lymphomas is multidisciplinary and requires the correlation between clinical, histopathological, immunohistochemical, and molecular diagnostic elements. In this article, we present four different cases of CD30-positive T-cell lymphoma with cutaneous manifestations. We compare cases with definitive diagnosis of papulosis lymphomatoid type C, primary cutaneous anaplastic large T-cell lymphoma, systemic anaplastic large T-cell lymphoma with secondary skin involvement, and mycosis fungoides with large cell transformation, highlighting the importance of clinicopathological correlation to classify these cases.
\end{abstract}

Keywords: Anaplastic large cell lymphoma; Cutaneous T-cell lymphoma; Lymphomatoid papulosis; Lymphoproliferative disorders; Mycosis fungoides; Primary cutaneous anaplastic large cell lymphoma

\section{INTRODUCTION}

Cutaneous lymphomas can be classified as primary when there is no involvement of extra-cutaneous body at the time of diagnosis; or as secondary; when cutaneous infiltration is caused by the dissemination of lymphoma originated in other organs. Among primary extranodal lymphomas, the incidence of primary cutaneous lymphomas (PCLs) is only smaller than gastrointestinal lymphomas. However, they are the most frequent type of T-cell lymphomas. It is crucial to distinguish PCLs from systemic analogs that can secondarily infiltrate the skin. They have different biological behavior, prognosis and therapeutic approach, despite the morphological similarities. PCLs are classified into cutaneous T-cell lymphomas (CTCLs) and cutaneous B-cell lymphomas, according to the source cell lineage. CTCLs correspond to $75 \%-80 \%$ of PCLs, and mycosis fungoides (MF) and its variants account for about $50 \%$ of PCLs. ${ }^{1,2}$

CD30-positive lymphoproliferative disorders that affect the skin are also (as the lymphomas in general) divided into primary and secondary. CD30-positive CTCLs account for about 30\% of CTCLs, setting the second most prevalent group within the diseases. ${ }^{3}$ CD30-positive CTCLs include: lymphomatoid papulosis (LP), anaplastic large cell lymphoma (ALCL), and some cases of mycosis fungoides with large cell transformation (MFLCT). ${ }^{3}$ Among the cutaneous CD30-positive lymphomas, we highlight the systemic anaplastic large T-cell lymphoma (SALCL), some cases of adult T-cell lymphoma/leukemia, and Hodgkin's lymphoma (HL). CD30 immunohistochemical expression is not specific for the above conditions, being necessary to point out that the classification of cutaneous lymphomas is only possible through the integration of clinical, histopathological, immunophenotypic, and molecular aspects. ${ }^{1,4}$

The present article reports four cases of CD30-positive lymphoproliferative diseases with skin involvement foccusing the exercise of differential diagnosis between these entities.

\section{CASE REPORTS}

Case 1

A 60-year-old female patient, asymptomatic and previously healthy. The patient have presented for 12 years cutaneous papules and erythematous-violet nodules. The lesions appeared in overlapping outbreaks, ulcerated, and resolved spontaneously, leaving residual atrophic scars. Affected areas included the trunk, buttocks, thighs, legs, arms, and forearms (Figure 1). The patient had already

Received on 05.06.2015

Approved by the Advisory Board and accepted for publication on 13.10.2015

Work performed at Hospital Federal de Bonsucesso and ID-Investigação em Dermatologia - Rio de Janeiro (RJ), Brazil.

Financial Support: None.

Conflict of Interest: None.

Hospital Federal de Bonsucesso - Bonsucesso (RJ), Brazil.

Instituto Nacional de Câncer (INCA) - Rio de Janeiro (RJ), Brazil.

C2017 by Anais Brasileiros de Dermatologia 
been submitted to four biopsies with inconclusive diagnoses. The patient showed good general condition through physical examination, without lymphadenopathy or hepatosplenomegaly. The biopsy of an infiltrated non-ulcerated papule revealed hyperkeratosis, rectification of the epidermis, and dense V-shaped infiltrate in the dermis, consisted of round cells with conspicuous cytoplasm and large pleomorphic vesicular nuclei, sometimes bevelled, displaying coarse nucleoli (Figure 2). Some binucleate cells resembling Reed-Sternberg cells, and numerous mitotic figures were observed. No intermingled neutrophils or eosinophils were identified. Immunohistochemistry showed positive reactions of neoplastic cells for anti-CD45LCA and anti-CD30, and negative for anti-CD3, anti-CD4, anti-CD8, and anti-CD20. After the final diagnosis of LP type C, treatment with UVB phototherapy was initiated.

\section{Case 2}

Patient 46-year-old male had reported the appearance of an erythematous nodule on the dorsum of the right foot four months before the appointment. The nodule evolved with progessive growth and ulceration. One month before, the patient presented with hardnodules on his right arm, leg and tight, following an upward path culminating in bulging in the ipsilateral inguinal region (Figure 3). Physical examination, laboratory test and CT scans of the chest, abdomen and pelvis showed no sistemic involvement. Anti-HIV and anti-HTLV serologies were negative. Skin biopsy revealed diffuse infiltration in the dermis, composed of rounded cells of conspicuous cytoplasm with large pleomorphic vesicular nuclei, sometimes cleaved, which allowed the visualization of one or more irregular nucleoli. Mitoses were easily observed (Figure 4). Immunohistochemistry resulted positive reaction for anti-CD45LCA, anti-CD3, anti-CD4, and anti-CD30, and negative for anti-CD20, anti-ALK (anaplastic large cell lymphoma kinase), anti-AE1/AE3, anti-Melan-A, anti-CD56, and anti-CD8. $80 \%$ of the cells were positive for Ki- 67.
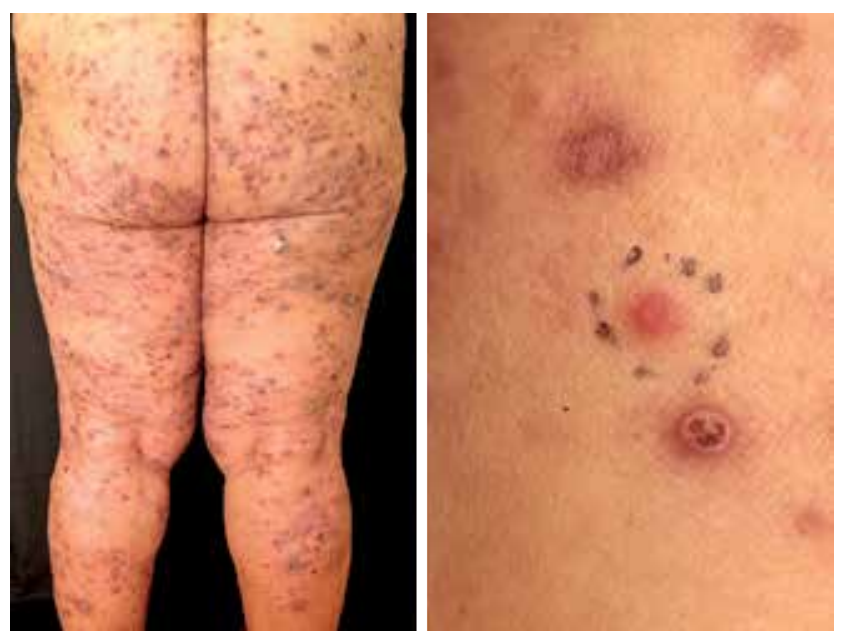

Figure 1: Lymphomatoid papulosis. A) Multiple erythematous papules, some with small erosions covered by scales and crusts and scarring lesions distributed symmetrically. B) Details of the lesions in three distinct evolutionary stages: the marking indicates a fully developed lesion (ideal for biopsy); under it, there is an ulcerated papule in involution; above, there is a completely regressed lesion
The final diagnosis was a primary cutaneous ALCL. The patient underwent chemotherapy with cyclophosphamide, doxorubicin, vincristine, and prednisone (CHOP) due to the topography and

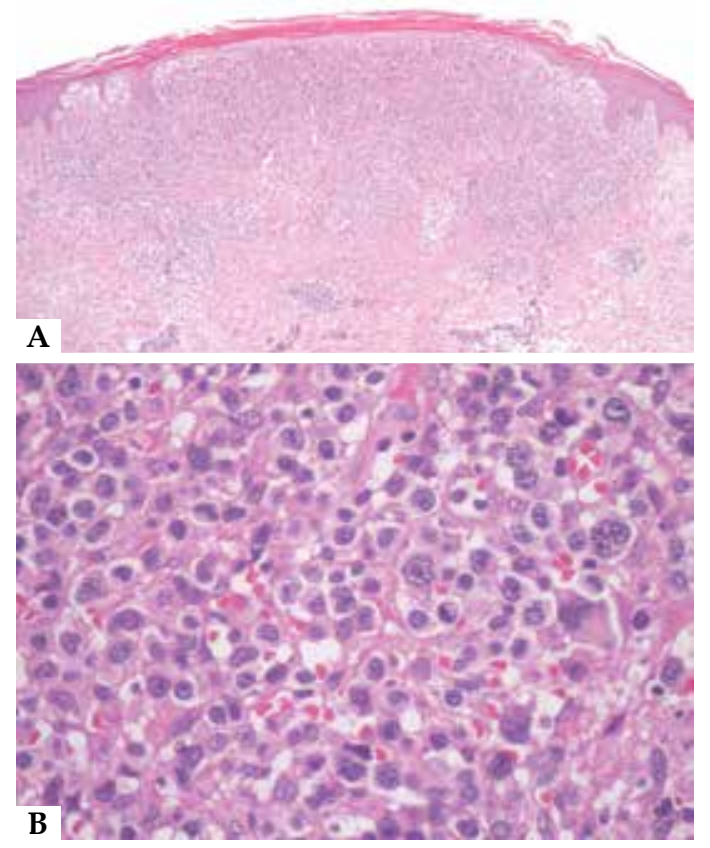

FIgURE 2: Lymphomatoid papulosis type C. A) Dermis showing a V-shaped cell infiltrate associated with blurring of the dermal-epidermal junction, rectification of epidermal cones, and hyperkeratosis (Hematoxylin \& eosin x40). B) Dense cellular infiltrate on the dermis, consisting of lymphocytes with large pleomorphic vesicular nuclei and conspicuous cytoplasm. Some binucleate lymphocytes resembling Reed-Sternberg cells are present (Hematoxylin \& $\operatorname{eosin} \mathrm{x} 400)$
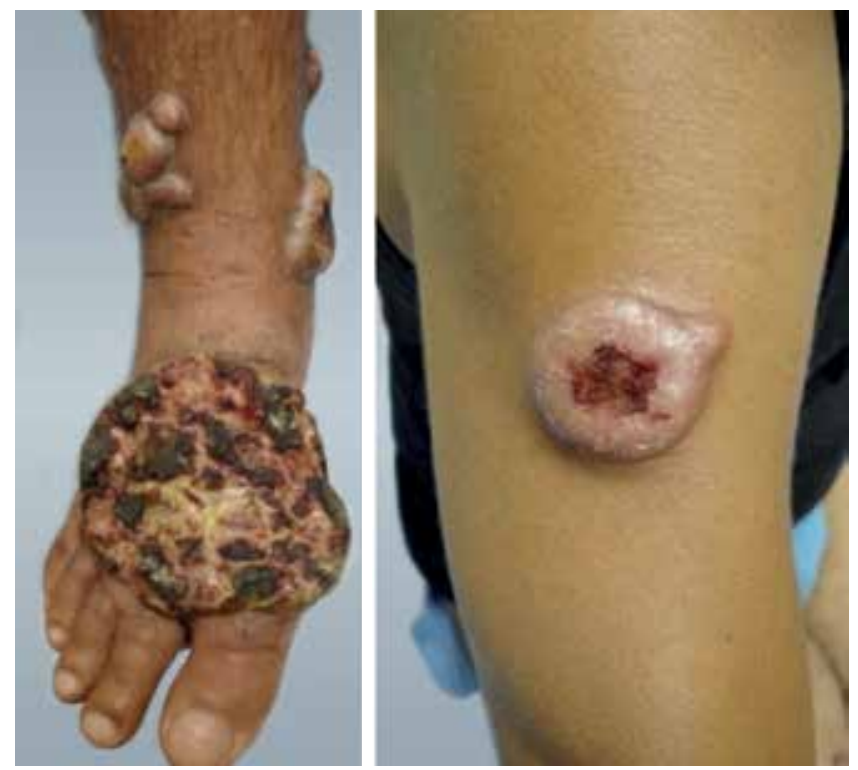

Figure 3: Primary cutaneous anaplastic large cell lymphoma. Nodular and tumor lesions with ulcerated surface present on the right foot, leg, and arm 


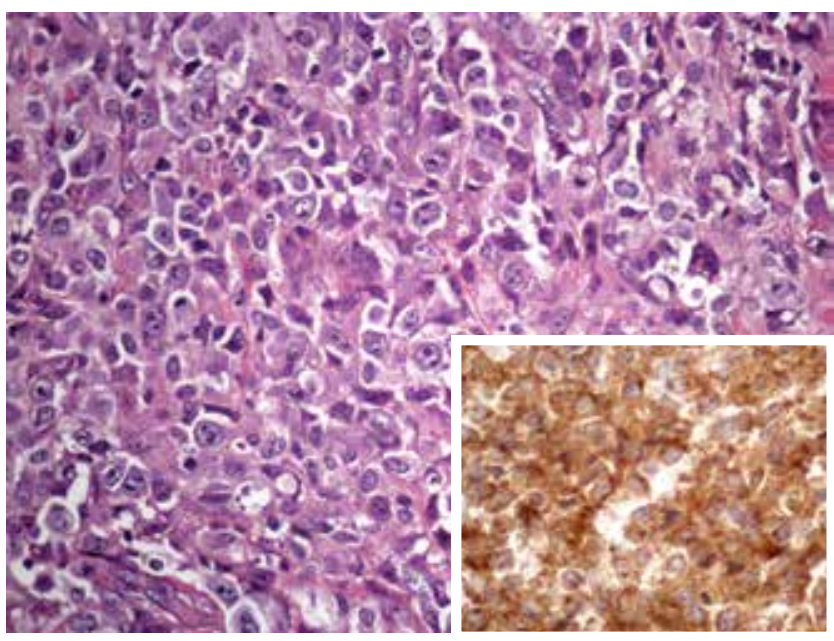

Figure 4: Primary cutaneous anaplastic large cell lymphoma. Dense diffuse infiltrate in the dermis, consisting of lymphocytes with large pleomorphic vesicular nuclei and conspicuous cytoplasm. Mitotic figures are easily visualized (Hematoxylin \& eosin x400). Inset: diffuse positivity of neoplastic lymphocytes in immunohistochemical reaction with anti-CD30 primary antibody (Hematoxylin \& eosin $x 400$ )

extent of the lesions, showing improvement. After seven months, the lesions recurred with lymph node involvement and evolution to death despite the attempt to treat them with other chemotherapy regimens.

\section{Case 3}

An 18-year-old male patient sought treatment with a clinical picture of pain and functional limitation of his right shoulder for one month associated with fever and weight loss. The patient was hospitalized and treated with intravenous antibiotic therapy for osteomyelitis. Dermatologic evaluation was requested due to a recent appearance of an ulcerated papule in the left scapular region (Figure 5). Laboratory tests showed normocytic and normochromic anemia, increased erythrocyte sedimentation rate, C-reactive protein, alkaline phosphatase, and gama-glutamyl transferase. Shoulder x-ray revealed lytic lesion of the right scapula. One month later, the lesion had increased and CT scan showed humerus lesions. A biopsy revealed ulcerated skin fragment with diffuse infiltration, consisting of lymphoid cells containing large vesicular nuclei, with abundant pale cytoplasm (Figure 6). Immunohistochemistry was positive for anti-CD30 and anti-ALK, with focal positivity for anti-CD3 and an approximate $30 \%$ positivity for anti-Ki67. Head, chest, abdomen, pelvis, and cervical CT scanning, and bone marrow biopsy showed no changes. Bone scintigraphy showed multiple areas of increased osteogenic activity, suggesting implants. Based on the results, the diagnosis was ALCL with cutaneous involvement. The patient was submitted to cycles of CHOP with etoposide, with shoulder pain improvement and disappearance of the cutaneous lesion in the first cycle. Remission was achieved and lesions have not recurred for the past four months.

\section{Case 4}

An 89-year-old female patient reported the appearance of nodular lesions on her left arm and in the posterior thoracic region, and a large eroded plaque with circinated edges in the right scapular region (Figure 7). Discreet erythema and scaling over the entire body surface were also noted. She reported having had asymptomatic or mildly pruritic intermittent erythematous scaly lesions 20 years before, which were diagnosed as skin allergy. Lymphadenopathy and hepatosplenomegaly were not observed. Skin biopsy revealed an infiltrate with a striated distribution in the papillary dermis and perivascular at depth, consisted of lymphoid cells of broad cytoplasm and large pleomorphic vesicular nuclei with one or more nucleoli (Figure 8). Some typical and atypical mitotic figures as well as mature lymphocytes and numerous eosinophils were found permeating the larger cells. Immunohistochemistry was positive for anti-CD3 and anti-CD30, and negative for anti-CD20, anti-CD4, anti-CD8, anti-CD56, and anti-EBV. The anti-Ki67 revealed a cell proli-
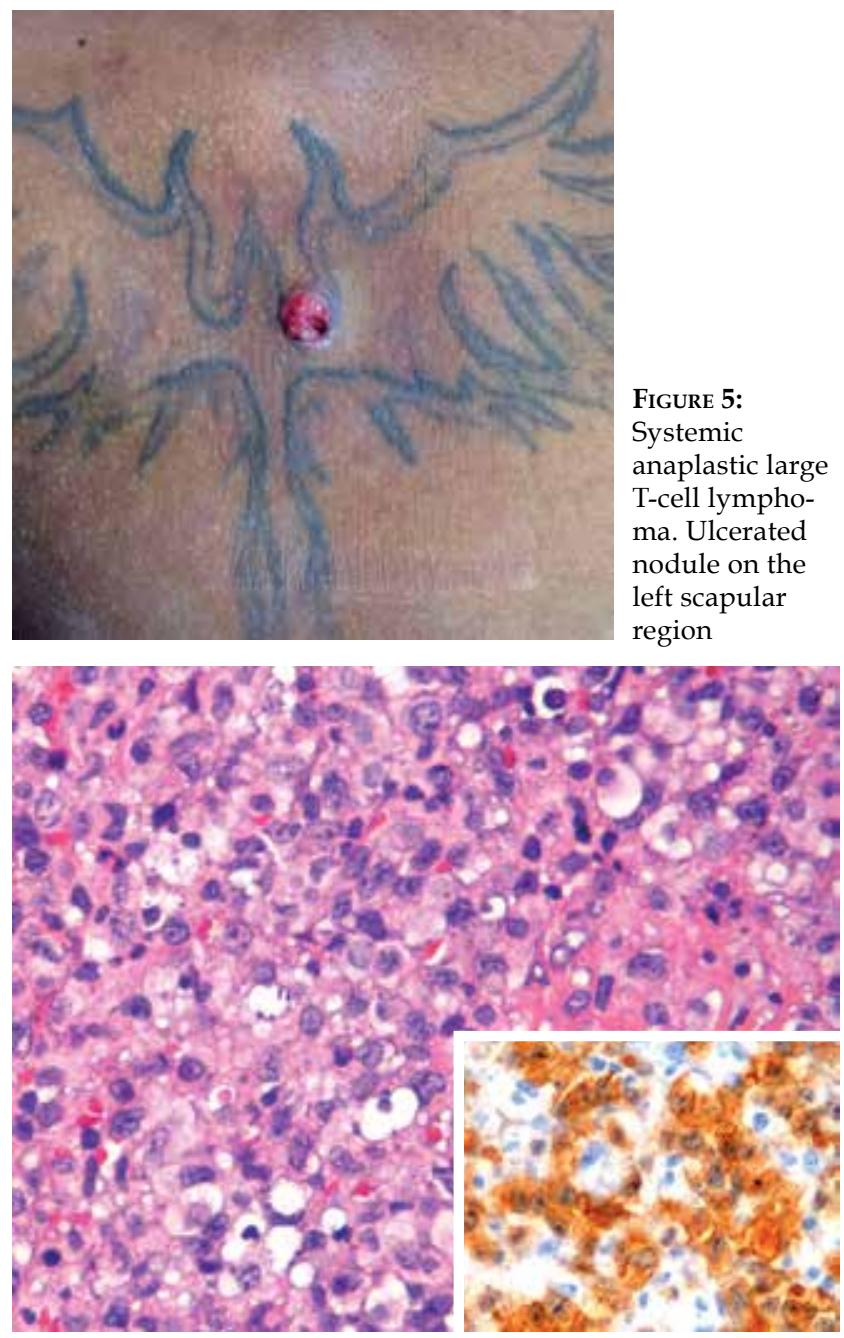

Figure 6: Systemic anaplastic large T-cell lymphoma. Dense diffuse infiltrate in the dermis, consisting of lymphocytes with large pleomorphic vesicular nuclei and conspicuous cytoplasm (Hematoxylin \& eosin $x 400$ ). Inset: diffuse positivity of neoplastic lymphocytes in immunohistochemical reaction with anti-ALK primary antibody (Hematoxylin \& eosin $x 400$ ) 


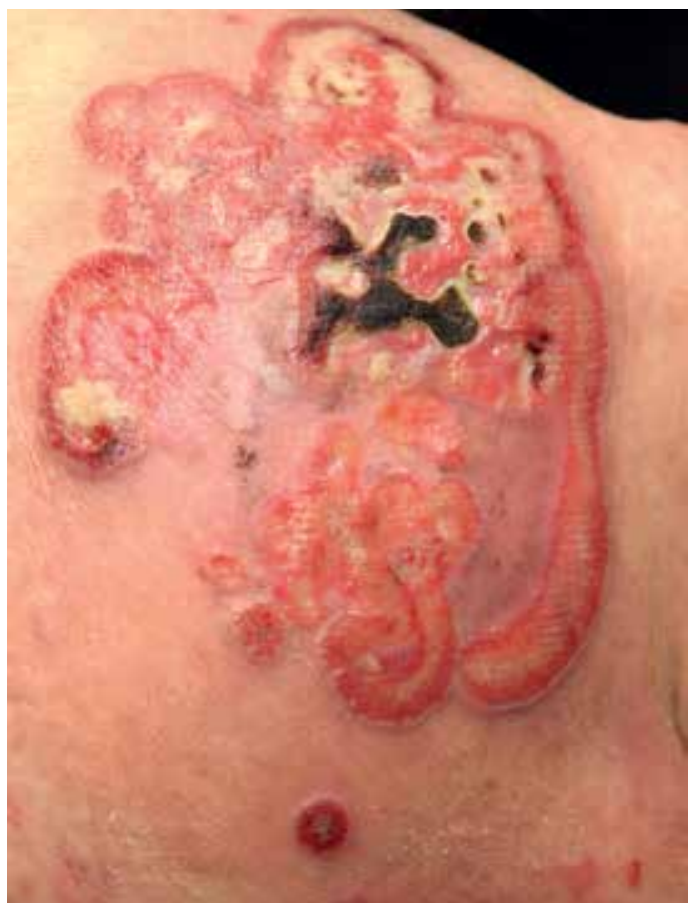

Figure 7: Large-cell transformation of mycosis fungoides. Plaque in polycyclic configuration with eroded surface. Ulceration covered by a blackened crust in the central portion. Note that the skin around the plaque reveals discreet erythema and fine scales that extended throughout the body

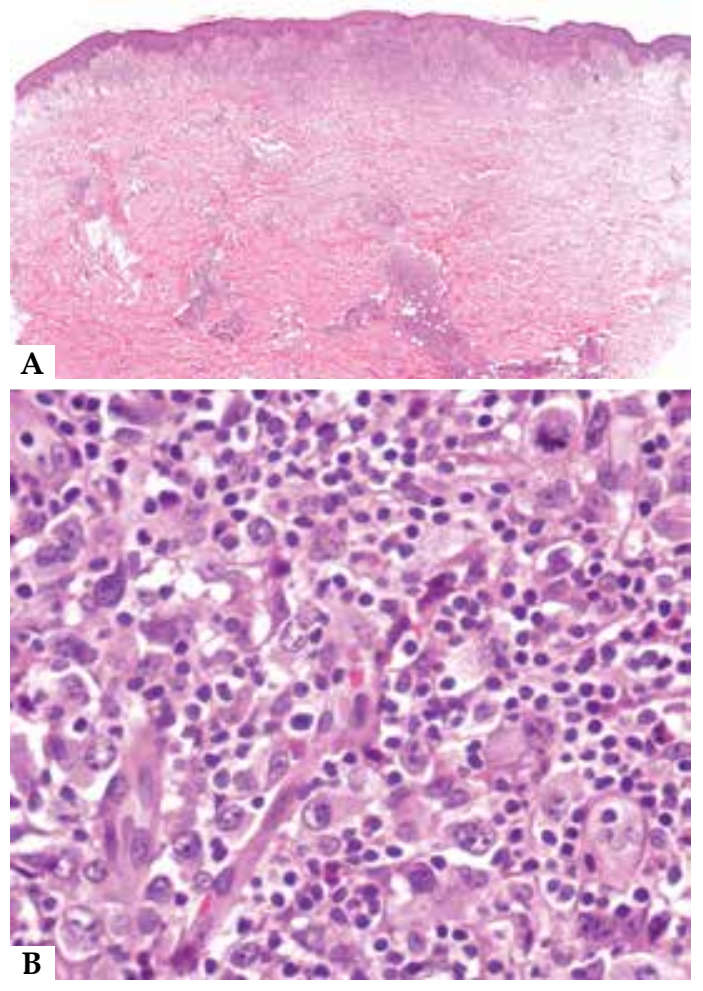

FIGURE 8: Large-cell transformation of mycosis fungoides. A) Dermis showing band-like infiltration in the papillary portion and around the skin appendages in depth (Hematoxylin \& eosin x20). B) Dermis showing lymphocytes with large pleomorphic vesicular nuclei and abundant cytoplasm, interspersed with small lymphocytes and eosinophils (Hematoxylin \& eosin x400) fieration index of $50 \%$. The diagnosis was MFLCT. The patient was treated with prednisone $20 \mathrm{mg} /$ day and methotrexate $10 \mathrm{mg} /$ week, with involution of the infiltrated lesions and re-epithelialization of erosions (in remission since August 2013).

\section{DISCUSSION}

The term lymphomatoid papulosis (LP) was first used by Macaulay in 1968 to describe a self-healing rhythmical paradoxical eruption, histologically malignant but clinically benign. Currently, LP is classified as a low-grade CTCL, in the spectrum of cutaneous CD30-positive lymphoproliferative disorders. ${ }^{4}$ The characteristic lesions are papules and eventually erythematous/coppery nodules, occurring in outbreaks. Lesions necrotize, ulcerate, and regress in within weeks. ${ }^{5}$ Outbreaks may overlap, causing lesions in different evolutionary stages, but the tendency to spontaneous resolution of each individual lesion is the hallmark of this dermatosis. Generally, lesions are widespread, with a predilection for the trunk and limbs. However, occurrences restricted to an anatomical area may occur. The number of lesions vary from a few to several hundred, and the symptoms can persist from months to decades. Lymphadenopathy and systemic involvement are not reported. ${ }^{5}$

Overall, LP is a chronic disease with an excellent prognosis. It may be associated with other lymphomas such as ALCL, MF or HL in up to $20 \%$ of the cases. ${ }^{4}$ Histopathologically, LP can present itself in different ways with four main recognized patterns. Its classical presentation (type A), the lymphocytes present large nuclei andconspicuous cytoplasm, sometimes binucleated, CD4 and CD30 positive are sparsely arranged amid V-shaped infiltration, rich in mature lymphocytes (reactive) eosinophils and neutrophils. The type B is characterized by small to medium CD4-positive lymphocytes and are often CD30-negative, with cerebriform nuclei and band-like (striated) arrangement in the superficial dermis with epidermotropism. These findings are indistinguishable from those found in MF. The type $\mathrm{C}$ has a dense monotonous population of large nuclei lymphocytes with conspicuous cytoplasm, CD4 and CD30-positivity, and no significant inflammatory background, resembling the presentation of an ALCL. The fourth proposed type, type $\mathrm{D}$, is characterized by medium to large-sized epidermotropic cell infiltrates, CD8+/CD30+, with minimal inflammatory background, simulating a primary cutaneous CD8-positive epidermotropic cytotoxic lymphoma. ${ }^{4}$ Therefore, the definitive diagnosis of LP requires close clinical-pathological correlation, especially when it comes to the least common histologic subtypes (B, C, and D). Case 1 was diagnosed as LP type $C$, with the essential documentation of minor lesions, outbreaks, and self-limited in differentiation with ALCL. The previous inconclusive biopsies from this patient stressed the need to correctly choose the lesion to be studied. It is necessary that the sample represent a recent and turgid lesion for proper histopathology yield, preferably without necrosis or ulceration. In case of regressive lesions, neoplastic cells generally undergo apoptosis, which prevents the specific diagnosis.

ALCL is a CD30-positive non-Hodgkin's lymphoma, classified by the World Health Organization as PCALCL and SALCL. ${ }^{5}$ ALCL affects more young adults and is two to three times more common in men. ${ }^{3}$ It often manifests itself as papules, nodules, or 
plaques. Lesions are usually solitary or few in number, generally restricted to a single anatomical area. However, it is estimated that $20 \%$ of patients have multifocal lesions. ${ }^{6}$ In contrast to LP lesions, PCALCL lesions tend to be fewer, larger, with slower and insidious onset, and usually do not regress spontaneously. The lesions are usually asymptomatic and systemic involvement is rare (which, by definition, cannot precede the appearance of cutaneous lesions). ${ }^{78} \mathrm{It}$ has a good prognosis with a 10-year survival rate of approximately $90 \%{ }^{8}$

Histopathology of PCALCLs shows evidence of diffuse dermal infiltrate of lymphoid cells containing large, round, oval, or irregular vesicular nuclei, with prominent and eosinophilic nucleoli and abundant cytoplasm. No epidermotropism is observed. ${ }^{3,7}$ Immunohistochemical study shows positivity for CD30 in more than $75 \%$ of tumor cells expressing T-cell markers (CD2, CD3, CD5, and CD7), generally T-helper cells (CD4). However, some cases may present memory CD8.,7 Unlike SALCL, cutaneous lymphoma expresses the cutaneous lymphocyte antigen (CLA), which determines tropism of the neoplastic cells to the skin, but does not express the epithelial membrane antigen (EMA) or ALK. ${ }^{3}$ Neoplastic cells do not express CD15, different from HL. ${ }^{3}$ Treatment consists of surgical excision (solitary lesion), radiation therapy, or low doses of methotrexate. ${ }^{3,7}$ Systemic chemotherapy is used in cases of rapidly progressive course or extracutaneous involvement, as happened with case $2 .{ }^{3,7}$ In a study of 135 patients with PCALCL, patients with leg involvement showed a 5-year survival rate of $76 \%$ for regional lesions and $67 \%$ for multifocal lesions, in contrast to a survival rate of $100 \%$ in patients without leg involvement. ${ }^{9}$ The evolution of case 2 supports a more aggressive behavior than expected for PCALCL with leg involvement, relapse, and death despite the appropriate chemotherapy regimen and close monitoring.

SALCL typically presents with painless lymphadenopathy and type B symptoms (fever, night sweats, or weight loss); $60 \%$ of patients have their disease diagnosed when it has already reached an advanced stage. Therefore, recommended treatment is polychemotherapy. ${ }^{10}$ Skin involvement occurs with the spread of the disease, featuring the IVD stage (Ann Arbor staging). SALCL cutaneous lesions show very similar morphology to PCALCL lesions. Therefore, it is essential to assess clinical course, staging, and immunohistochemical differentiation between these two lymphomas with distinct biological behavior. ${ }^{10}$ SALCL is associated with translocation $(2 ; 5)$ in $60 \%$ of cases, resulting in the expression of ALK protein. ${ }^{11}$ In its turn, since PCALCL is not related to this translocation, it does not express ALK. ${ }^{8}$ ALK protein is a marker of good prognosis in SALCLs, with a 5-year survival rate of $71 \%-100 \%$, while in ALK-negative SALCLs, the survival rate is $15 \%-45 \%{ }^{11}$ ALK-positive SALCLs affect younger men in the fourth decade of life, while ALK-negative are more frequent in older patients. ${ }^{7,10}$ Recently, brentuximab vedotin - an anti-CD30-auristatin-E/monomethyl antibody-drug conjugate - has been approved for use in relapsed CD30-positive HL and SAL-
CL patients, based on excellent responses in phase II studies. ${ }^{12}$ The use with CD30-positive PCLs patients looks promising, but further studies are needed to establish its indication to clinical practice. ${ }^{13-15}$

MF presents itself initially as patches or plaques that can develop into a tumor over the years. ${ }^{16,17}$ Histopathologicaly, the patchy lesions show dense band-like infiltrate in the upper portion of the dermis, consisting of small lymphocytes with cerebriform nuclei, which align along the dermal-epidermal junction and permeate the Malpighian layer in the absence of significant spongiosis (epidermotropism). ${ }^{18}$ It expresses T-cell receptors, being CD4-positive/CD8-MF-negative the most common phenotypes. ${ }^{11}$ As MF progresses to the tumor stage, the infiltration of neoplastic lymphocytes takes a nodular pattern in the dermis with progressive loss of epidermotropism, which can eventually become absent, complicating the differential diagnosis. In $50 \%$ of patients with tumor stage $\mathrm{MF}$, progression to large cells may occur, defined by more than $25 \%$ of large lymphoid cells in the infiltrate or by the formation of small nodular groups of large cells within the infiltrate. ${ }^{17}$ Large-cell transformation worsens the prognosis and requires more aggressive treatment, with a mean survival rate of less than two years. ${ }^{17,19}$ Some MFLCT cases may present CD30 expression by more than $10 \%$ of tumor cells. The 5-year survival rate of patients with MFLCT is $11 \%$ $32 \%$, and the group expressing CD30 has a better prognostic. ${ }^{20}$

Histopathological differential diagnosis between MFLCT, ALCL, and LP type C can be quite difficult, but we can consider MFLCT more likely when cerebriform lymphocytes coexist with large lymphocytes, when CD30 expression occurs in less than 75\% of large cells, or when there is foliculotropism or epidermotropism. ${ }^{18}$ It should be emphasized that the definitive diagnosis of MFLCT requires the identification through clinical history or evolutionary follow-up of the classic MF patchy lesions for long periods prior to the development of tumors (clinical assessment) and histopathological examination of the cutaneous infiltration by large atypical lymphocytes.

MFLCT treatment is a challenge, as it often presents a poor response. The prognosis is particularly poor in patients who have several sites with transformation. In younger patients, the treatment is done with radiation therapy (unifocal transformation) or systemic chemotherapy. In older patients with unifocal transformation, radiotherapy can also be used and occasionally leads to long-term remissions. ${ }^{19}$ In case 4 , physicians opted for conservative treatment with prednisone and low doses of methotrexate due to the age of the patient, with excellent results.

In this article, we presented 4 different cases of CD30-positive T-cell lymphoma. We emphasize the need for close clinicopathologic correlation for the definitive diagnosis of these lymphomas, highlighting the participation of dermatologists. In case 3, it would be possible to favor a diagnosis of SALCL on pathological grounds due to the positivity for ALK. On the other hand, if clinical and evolutionary evaluation of patients of the other cases were not available, it would not be possible to go beyond a descriptive diagnosis of "cutaneous CD30-positive T-cell lymphoma. $]$ 


\section{REFERENCES}

1. Willemze R, Hodak E, Zinzani PL, Specht L, Ladetto M; ESMO Guidelines Working Group. Primary cutaneous lymphomas: ESMO Clinical Practice Guidelines for diagnosis, treatment and follow-up. Ann Oncol. 2013;24:vi149-54.

2. Yamashita T, Abbade LPF, Marques MEA, Marques SA. Mycosis fungoides and Sézary syndrome: clinical, histopathological and immunohistochemical review and update. An Bras Dermatol. 2012;87:817-30.

3. Sanches Jr JA, Moricz CZM, Neto CF. Lymphoproliferative processes of the skin. Part 2 - Cutaneous T-cell and NK-cell lymphomas. An Bras Dermatol. 2006;81:725

4. McQuitty E, Curry JL, Tetzlaff MT, Prieto VG, Duvic M, Torres-Cabala C. The differential diagnosis of CD8-positive ("type D") lymphomatoid papulosis. J Cutan Pathol. 2014;41:88-100.

5. Akilov OE, Pillai RK, Grandinetti LM, Kant JA, Geskin L. Clonal T-Cell receptor $\gamma$-chain gene rearrangements in differential diagnosis of Lymphomatoid Papulosis from skin metastasis of Nodal Anaplastic Large-Cell Lymphoma. Arch Dermatol. 2011;147:943-7.

6. Kumaran MS, Jithendriya M, Nagaraj P, Tirumalae R, Jayaseelan E. Anaplastic lymphoma kinase-positive primary cutaneous anaplastic large cell lymphoma- Is it a new variant? Indian J Dermatol Venereol Leprol. 2012;78:354-7.

7. Oliveira LS, Nobrega MP, Monteiro MG, Almeida WL. Primary cutaneous anaplastic large-cell lymphoma - Case report. An Bras Dermatol. 2013;88:132-5

8. Benner MF, Willemze R. Bone marrow examination has limited value in the staging of patients with an anaplastic large cell lymphoma first presenting in the skin. Retrospective analysis of 107 patients. Br J Dermatol. 2008;159:1148-51.

9. Benner MF, Willemze R. Applicability and Prognostic Value of the New TNM Classification System in 135 Patients With Primary Cutaneous Anaplastic Large Cell Lymphoma. Arch Dermatol. 2009;145:1399-404.

10. Bird JE, Leitenberger JJ, Solomon A, Blauvelt A, Hopkins S. Fatal ALK-negative systemic anaplastic large cell lymphoma presenting with disseminated cutaneous dome-shaped papules and nodules. Dermatol Online J. 2012;18:5.

11. Kempf W, Kazakov DV, Kerl K. Cutaneous lymphomas. An update. Part 1: T-cell and natural killer/ T-cell lymphomas and related conditions. Am J Dermatopathol. 2014;36:105-23.

12. van de Donk NW, Dhimolea E. Brentuximab vendoitin. MAbs. 2012;4:458-65.

13. Criscuolo M, Fianchi L, Voso MT, Pagano L. Rapid response of nodular CD30positive mycosis fungoides to brentuximab vedotin. Br J Haematol. 2015;168:617.

14. Mody K, Wallace JS, Stearns DM, Bowers G, Lacy SR, Levy NB, et al. CD30positive cutaneous T-cell lymphoma and response to Brentuximab Vedotin: 2 illustrative cases. Clin Lymphoma Myeloma Leuk. 2013;13:319-23.

15. Mehra T, Ikenberg K, Moos RM, Benz R, Nair G, Schanz U, et al. Brentuximab as a treatment for CD30+ mycosis fungoides and Sézary syndrome. JAMA Dermatol. 2015;151:73-7.

16. Ackerman $A B$, Jeunon T, Millete F. Advanced dermatopathology: Smallplaque parapsoriasis, like large-plaque parapsoriasis, is mycosis fungoides. Dermatopathol: Pract \& Conc. 2009;15:6

17. Chandra P, Plaza JA, Zuo Z, Diwan AH, Koeppen H, Duvic M, et al. Clusterin expression correlates with stage and presence of large cells in mycosis fungoide. Am J Clin Pathol. 2009;131:511-5.

18. Barberio E, Thomas L, Skowron F, Balme B, Dalle S. Transformed mycosis fungoides: clinicopathological features and outcome. $\mathrm{Br} \mathrm{J}$ Dermatol. 2007;157:284-9.

19. Prince HM, Whittaker S, Hoppe RT. How I treat mycosis fungoides and Sézary syndrome. Blood. 2009;114:4337-53.

20. Benner MF, Jansen PM, Vermeer MH, Willemze R. Prognostic factors in transformed mycosis fungoides: a retrospective analysis of 100 cases. Blood. 2012;119:1643-9.
MAILING ADDRESS:

Samira Barroso Jorge

Av. Londres, 616 - Prédio 6

Bonsucesso

21041-030 Rio de Janeiro, RJ - Brazil

E-mail:samirabarroso@yahoo.com.br

How to cite this article: Jeunon T, Jorge SB, Gonzaga YBM. CD30-positive cutaneous lymphoma: report of four cases with an emphasis on clinicopathological correlation. . An Bras Dermatol. 2017;92(1):86-91. 